\title{
Eimeria bovis-triggered neutrophil extracellular trap formation is CD11b-, ERK 1/2-, p38 MAP kinase- and SOCE-dependent
}

Tamara Muñoz-Caro ${ }^{1 *}$, Sandra Jaqueline Mena Huertas ${ }^{2,3}$, Ivan Conejeros ${ }^{2}$, Pablo Alarcón², María A Hidalgo², Rafael A Burgos ${ }^{2}$, Carlos Hermosilla' and Anja Taubert ${ }^{1}$

\begin{abstract}
Eimeria bovis is an important coccidian parasite that causes high economic losses in the cattle industry. We recently showed that polymorphonuclear neutrophils (PMN) react upon E. bovis sporozoite exposure by neutrophil extracellular trap (NET) formation. We focused here on the molecular mechanisms that are involved in this process. The sporozoite encounter led to an enhanced surface expression of neutrophil CD11b suggesting a potential role of this receptor in E. bovis-mediated NETosis. Antibody-mediated blockage of CD11b confirmed this assumption and led to a significantly decreased sporozoite-triggered NET. In addition, E. bovis-induced NETosis was found to be $\mathrm{Ca}^{2+}$-dependent since the inhibition of store-operated calcium entry (SOCE) significantly diminished NET. Furthermore, NADPH oxidase, neutrophil elastase (NE) and myeloperoxidase (MPO) were confirmed as key molecules in sporozoite-triggered NETosis, as inhibition thereof blocked parasite-triggered NET. PMN degranulation analyses revealed a significant release of matrix metalloprotease-9 containing granules upon sporozoite exposure. We further show a significantly enhanced phosphorylation of ERK1/2 and P38 MAPK in sporozoite-exposed PMN indicating a key role of this signaling pathway in E. bovis-mediated NETosis. Accordingly, ERK 1/2 and p38 MAPK inhibition led to a significant decrease in NET formation. Finally, we demonstrate that sporozoite-induced NETosis is neither a stage-, species-, nor host-specific process.
\end{abstract}

\section{Introduction}

Eimeria bovis is an important intracellular apicomplexan parasite of cattle, causing severe haemorrhagic diarrhoea (typhlocolitis) especially in calves leading to high economic losses worldwide [1]. Polymorphonuclear neutrophils (PMN) appear to play a pivotal role in E. bovis defense. This leukocyte population was identified in parasitized intestine of E. bovis-infected calves [2] and PMN have been shown to interact directly with $E$. bovis stages and antigen, resulting in direct elimination or production of pro-inflammatory cytokines, chemokines and iNOS upon encounter [3]. Additionally, PMN were shown to adhere to E. bovis-infected host endothelial cells [4] and their phagocytic and oxidative burst activities were enhanced in response to $E$. bovis sporozoites in vitro or ex vivo during

\footnotetext{
* Correspondence: Tamara.Munoz-Caro@vetmed.uni-giessen.de 'Institute of Parasitology, Justus Liebig University Giessen, Schubertstraße 81, 35392 Giessen, Germany

Full list of author information is available at the end of the article
}

infection [3]. Furthermore, we recently identified sporozoites of E. bovis as potent inducers of neutrophil extracellular traps (NET) [5]. Overall, NET formation has been described as a novel form of cell death called ETosis which is distinct from apoptosis, autophagy and necrosis leading to extracellular entrapment and eventually the killing of pathogens $[6,7]$. NET have been shown to be involved in several infections caused by bacteria, viruses and fungi [8-11]. However, little attention has been paid to the role of NET in the early host innate immune response against protozoan parasites [12,13]. By now, NET formation has been described to be induced by some protozoan parasites, such as Leishmania spp. [14], E. bovis, Toxoplasma gondii, Plasmodium falciparum, E. arloingi, and Besnoitia besnoiti [5,12,15-17].

NETosis is an NADPH oxidase-mediated ROS-dependent mechanism [7-11] which causes the expulsion of a mixture of nuclear and cytoplasmic granule contents leading to the formation of fiber-like structures being decorated with 
histones and granular molecules, such as neutrophil elastase (NE) and myeloperoxidase (MPO) amongst others [7,10,11]. On the mechanistic level, parasites are immobilized within these DNA-rich structures and may be killed via high local concentrations of antimicrobial molecules [12,13]. Consequently, some cases of parasite-triggered NETosis have been described as ROS-, NE- or MPO-dependent [5,12,16,17].

So far, few pathogen-derived molecules triggering NETosis have been identified such as bacterial toxins [18], virus [19] or surface lipophosphoglycans of Leishmania [14]. In addition, little data are reported on NET-associated PMNderived ligands and $\mathrm{Ca}^{2+}$-mediated signalling pathways [20]. As such, CD11b and CD18 are described as being involved in Candida albicans [21] or Mannheimia haemolyticamediated NETosis [18]. Recently $\mathrm{Ca}^{2+}$-dependent ETosis has been reported [20]. In the case of parasite-triggered NETosis, no data on PMN ligands or $\mathrm{Ca}^{2+}$-dependency are available, so far. This work focuses on molecular mechanisms involved in $E$. bovis-induced NET formation. We here demonstrate that respective NETosis depends on granulocyte NE and MPO activities and on the activation of an ERK1/2- and p38-related signaling pathway. Furthermore store-operated $\mathrm{Ca}^{2+}$ entry and CD11b receptor binding is involved in E. bovis-triggered NETosis.

\section{Materials and methods Parasite}

The E. bovis (strain $\mathrm{H}$ ) used in the present study was maintained by passages in Holstein-Friesian calves for oocyst production as described by Hermosilla et al. [22]. Calves were infected orally with $5 \times 10^{4}$ sporulated $E$. bovis oocysts. Collection of oocysts, oocyst sporulation and excystation of sporozoites were performed as previously described [22]. Free-released sporozoites were washed three times with sterile PBS $(400 \times g, 10 \mathrm{~min})$, counted in a Neubauer haemocytometer chamber and thereafter suspended at final concentrations of $2.5 \times 10^{5}-10^{6}$ sporozoites/mL in culture medium (RPMI 1640, 1\% penicillin/ streptomycin, v/v; Gibco) free of fetal calf serum (FCS) until further experimental use. In addition, sporulated E. arloingi oocysts (strain A) [16], Isospora suis oocysts (kindly provided by Anja Joachim, University of Veterinary Medicine of Vienna, Austria) and Toxoplasma gondii oocysts (kindly provided by Anja Joachim, University of Veterinary Medicine of Vienna, Austria and Peter Deplazes, Faculty of Veterinary Medicine, University of Zurich, Switzerland) were used for comparative analyses on parasite speciesspecific NET induction. Sporozoite excystation of the latter parasite species was performed according to Pinckney et al. [23] as well as Freyre and Falcon [24].

\section{Isolation of bovine PMN}

Adult dairy cows $(n=3)$ were bled by puncture of the jugular vein and blood was collected in $50 \mathrm{~mL}$ plastic tubes (Nunc) containing $0.1 \mathrm{~mL}$ heparin (Sigma-Aldrich) as anticoagulant. Heparinized blood was diluted in an equal amount of PBS containing 0.02\% EDTA (SigmaAldrich), layered on Biocoll Separating Solution (Biochrom AG) and centrifuged at $800 \times g$ for $45 \mathrm{~min}$. After removal of the plasma and PBMC layer, the pellet was suspended in $25 \mathrm{~mL}$ distilled water and gently shaken for $40 \mathrm{~s}$ to lyse erythrocytes. Osmolarity was immediately re-adjusted by adding $3 \mathrm{~mL}$ of Hanks Salt Solution (HBSS $10 \mathrm{x}$, Biochrom AG). PMN were washed twice, re-suspended in RPMI 1640 medium (Gibco), counted in a Neubauer haemocytometer chamber and subsequently incubated at $37{ }^{\circ} \mathrm{C}$ and $5 \% \mathrm{CO}_{2}$ atmosphere for at least $30 \mathrm{~min}$ before use.

\section{Flow cytometric analysis of Eimeria bovis sporozoites-induced CD11b surface expression on bovine PMN}

PMN were incubated with CFSE-stained E. bovis sporozoites (1:1 ratio, $\left.30 \mathrm{~min}, 37{ }^{\circ} \mathrm{C}\right)$ in HBSS containing $0.9 \mathrm{mM} \mathrm{CaCl}_{2}$. For positive controls, PMN were treated with platelet activating factor (PAF $100 \mathrm{nM}, 15 \mathrm{~min}, 37^{\circ} \mathrm{C}$; Calbiochem). For negative controls, non-treated and nonexposed PMN were used. After incubation, cells were pelleted $\left(300 \times g, 20^{\circ} \mathrm{C}, 6 \mathrm{~min}\right)$, resuspended in $200 \mu \mathrm{L}$ HBSS and incubated with anti-CD11b antibodies coupled to allophycocyanin (APC; clone M1/70; $20 \mathrm{~min}$, in the dark; BD Pharmingen) and washed at $300 \times g, 20{ }^{\circ} \mathrm{C}, 6 \mathrm{~min}$. Thereafter, the cells were analyzed using a FACS Canto II cytometer (BD Biosciences, San Diego, CA, USA). The data were displayed as plots of forward versus side light scatter. The mean fluorescence of APC was determined from a minimum of $10^{4}$ cells using BD FACS Diva 6.1 software (BD Biosciences, San Diego, CA, USA).

\section{Inhibition of store-operated calcium entry (SOCE)}

Bovine PMN were pre-incubated with the SOCE inhibitor 2-aminoethoxydiphenyl borate (2-APB) for $15 \mathrm{~min}$ at concentrations of 50 and $100 \mu \mathrm{M}$ in HBSS medium prior to exposure to E. bovis sporozoites in a $1: 2$ ratio $\left(2 \times 10^{5}\right.$ PMN: $4 \times 10^{5}$ sporozoites, $60 \mathrm{~min}, 37^{\circ} \mathrm{C}$ ).

For NET quantification, $50 \mu \mathrm{L}$ of micrococcal nuclease buffer containing $0.1 \mathrm{U} / \mu \mathrm{L}$ micrococcal nuclease (both New England Biolabs) were added to each well and incubated $\left(15 \mathrm{~min}, 37^{\circ} \mathrm{C}\right)$. Afterwards the samples were centrifuged $(300 \times g, 5 \mathrm{~min})$ and the supernatants were transferred into a 96-well flat-bottom plate $(100 \mu \mathrm{L}$ per well in duplicates). Fifty microliters of Pico Green ${ }^{\circ}$ (1:200 dilution in $10 \mathrm{mM}$ Tris base buffered with $1 \mathrm{mM}$ EDTA) were added to each sample and the samples were incubated (4 min, in the dark). NET formation was determined by spectrofluorometric analysis at an excitation wavelength of $484 \mathrm{~nm}$ and an emission wavelength of $520 \mathrm{~nm}$ using an automated plate monochrome reader (Varioskan Flash ${ }^{\circledR}$; Thermo Scientific). For negative controls, 
PMN in plain medium were used. For positive controls, stimulation with zymosan $(1 \mathrm{mg} / \mathrm{mL})$ was used.

\section{Immunoblotting for the detection of phosphorylated ERK $1 / 2$ and $\mathrm{p} 38 \mathrm{MAPK}$}

PMN $\left(5 \times 10^{6}\right.$ in HBSS containing $\left.0.9 \mathrm{mM} \mathrm{CaCl}_{2}\right)$ were exposed to E. bovis-sporozoites $\left(5 \times 10^{6}\right)$ for 15 and $30 \mathrm{~min}$ at $37{ }^{\circ} \mathrm{C}$ in a final volume of $500 \mu \mathrm{L}$. For positive controls, PMN were stimulated with PAF (100 nM). Thereafter, total protein was extracted as described previously by Hidalgo et al. [25] and $40 \mu \mathrm{g}$ total protein were analyzed by a $10 \%$ SDS-PAGE. Immunoblotting was performed according to a protocol previously described by Hidalgo et al. [26]. Antibodies directed against phosphop38 MAPK and phospho-ERK1/2 (Cell Signalling, Beverly, MA, USA) were used according to the instructions provided by the manufacturer. Anti-mouse HRP-conjugated antisera (Santa Cruz Biotechnology, USA) were used as secondary antibodies (2 h of incubation, RT, in constant agitation). Signals were detected using an enhanced chemiluminescence system (Western Lightning ${ }^{\bullet}$ PlusECL; Perkin-Elmer, USA). After signal detection and documentation, the bound antibodies were removed by stripping the membranes (100 mM 2-mercaptoethanol; 2\% SDS; $62.5 \mathrm{mM}$ Tris- $\mathrm{HCl}, \mathrm{pH} 6.7$, for $2 \mathrm{~h}$ at $50{ }^{\circ} \mathrm{C}$ with agitation, followed by several washes with TBS-Tween $0.1 \%$ ) according to Hidalgo et al. [25] and each membrane was re-probed with an antibody recognizing total p38 MAPK (p38 MAPK anti rabbit antibody; Cell signaling technology) and ERK1/2 (rabbit polyclonal IgG; Santa Cruz Biotechnology, USA). The samples were then further processed as described above. The intensities of each band were analyzed using the Software Image J and the signals were normalized to total ERK1/ 2 for p-ERK1/2 and to total p38 for p-p38.

\section{NET inhibition assays using ERK1/2, p38 MAPK inhibitors} and functional CD11b monoclonal antibodies

For the inhibition of ERK 1/2 and p38 MAPK the following inhibitors were used respectively: UO126 $(50 \mu \mathrm{M}$; Sigma-Aldrich) and SB 203580 (10 $\mu$ M; Sigma-Aldrich). Therefore, PMN were preincubated with the inhibitors for $30 \mathrm{~min}$ at RT in HBSS-buffer without phenol red (Gibco). $\mathrm{CD} 11 \mathrm{~b}$ was blocked via pre-incubation in mouse anti bovine CD11b monoclonal antibodies (MCA1425, diluted 1:5 in PBS; AbD Serotec). For antibody control, we used an irrelevant antibody at an identical concentration (anti-bovine CD4, AbD Serotec). Then, PMN were exposed to viable $E$. bovis-sporozoites (1:2 ratio: $2 \times 10^{5}$ $\mathrm{PMN}+4 \times 10^{5}$ sporozoites) for $60 \mathrm{~min}$ at $37{ }^{\circ} \mathrm{C}$. Thereafter extracellular DNA was quantified as described above. All measurements were achieved using an automated monochrome reader (Varioskan Flash; Thermo Scientific). For positive controls, zymosan (Sigma-Aldrich) was used in a final concentration of $0.5 \mathrm{mg} / \mathrm{mL}$.
Visualization of NET and detection of histones (H3), neutrophil elastase (NE) and myeloperoxidase (MPO) in NET structures

Bovine PMN were incubated with E. bovis sporozoites (ratio 1:1; $30 \mathrm{~min}$ ) on poly-${ }_{-}$-lysine-treated coverslips and fixed $[4 \%(\mathrm{w} / \mathrm{v})$ paraformaldehyde, Merck, $20 \mathrm{~min}$ in the dark]. NET structures were visualized by staining extracellular DNA with $5 \mathrm{mM}$ Sytox Orange dye (Invitrogen) for $10 \mathrm{~min}$ at RT according to Martinelli et al. [27]. For the visualization of sporozoites within NET structures, sporozoites were stained with 5(6)-carboxyfluorescein diacetate succinimidyl ester (CFSE, $7.5 \mu \mathrm{M}, 37^{\circ} \mathrm{C}$, $30 \mathrm{~min}$; Invitrogen) according to Hermosilla et al. [28] prior to PMN exposure. After fixation of the cells and three washings in sterile PBS, the samples were mounted in anti-fading buffer (Mowiol ${ }^{\circ}$, Sigma-Aldrich) and stored $\left(4^{\circ} \mathrm{C}\right.$, in the dark) until further use. For the detection of histones, MPO and NE within NET structures the following specific antibodies were used: anti-histone monoclonal antibodies [rabbit (E173) anti-bovine histone H3, phospho S10 DyLight $^{\circ}$ 488, 1:100; ab139848, Abcam], anti-MPO antibodies (rabbit anti-bovine MPO, Alexa Fluor 488, 1:200; ABIN906866) and anti-NE antibodies (rabbit anti-human NE, 1:200; AB68672, Abcam). Therefore, the samples were washed three times with PBS, blocked with BSA $[1 \%(\mathrm{w} / \mathrm{v})$ in PBS, 30 min, RT, Sigma-Aldrich] and reacted with antihistone, anti-NE or anti-MPO antibodies $[1 \mathrm{~h}, \mathrm{RT}$, in the dark for bovine anti-histone (H3); $24 \mathrm{~h}$, RT, in the dark for both anti-MPO and anti-NE antibodies]. The samples were then gently washed in PBS and mounted in antifading buffer (Mowiol ${ }^{\circ}$, Sigma-Aldrich). Visualization was achieved using an inverted Olympus ${ }^{\circ}$ IX81 fluorescence microscope equipped with a digital camera.

Measurements of NADPH oxidase, NE and MPO enzymatic activities and inhibition of these enzyme activities

For NADPH oxidase-, NE- and MPO-inhibition assays, the following inhibitors were used: the NADPH oxidase inhibitor diphenylene iodonium (DPI, $10 \mu \mathrm{M}$, SigmaAldrich); the NE inhibitor Suc-Ala-Ala-Pro-Val chloromethyl ketone (CMK; $1 \mathrm{mM}$, Sigma-Aldrich), according to Scapinello et al. [29] and the MPO inhibitor 4-aminobenzoic acid hydrazide (ABAH; $100 \mu \mathrm{M}$, Calbiochem), according to Parker et al. [30]. In brief, PMN and sporozoites (1:1 ratio, $n=3$ ) were incubated ( $30 \mathrm{~min}, 37^{\circ} \mathrm{C}$ ) in HBSS-buffer without phenol red (Gibco) for positive controls. In parallel, PMN were pre-incubated with the corresponding inhibitors for $30 \mathrm{~min}$ at $\mathrm{RT}$ prior to exposure to viable E. bovis-sporozoites (1:1 ratio, $\left.30 \mathrm{~min}, 37^{\circ} \mathrm{C}\right)$. To estimate maximum values of extracellular DNA, PMN were lysed by Triton-X 100 treatment (0.1\%; Sigma-Aldrich). To block NET formation, $90 \mathrm{U}$ of DNase I (Roche Diagnostics) were supplemented 15 min before the end of incubation period. NET were quantified via Pico Green DNA staining as described above. 
NADPH oxidase, NE and MPO enzymatic activities were measured using respective substrates: $10 \mu \mathrm{g} / \mathrm{mL}$ DCFH-DA (Sigma-Aldrich); $3 \mathrm{mg} / \mathrm{mL}$ of the NE chromogenic substrate MeoSuc-Ala-Ala-Pro-Val-chloromethyl-ketone (SigmaAldrich) and $50 \mu \mathrm{M}$ Amplex red (Invitrogen), respectively. ROS production was measured by oxidation of DCFH-DA to fluorescent DCF according to Conejeros et al. [31]. The relative fluorescence units (RFU) were recorded at $15 \mathrm{~min}$ intervals for a period of $30 \mathrm{~min}$ applying $485 \mathrm{~nm}$ excitation and $530 \mathrm{~nm}$ emission wavelengths. NE activity was measured every 10 min for $30 \mathrm{~min}$ at $410 \mathrm{~nm}$ wavelength and MPO-peroxidase activity was measured every $10 \mathrm{~min}$ for $30 \mathrm{~min}$ in 571 $585 \mathrm{~nm}$ fluorescence ranges. All measurements were achieved using an automated monochrome reader (Varioskan Flash; Thermo Scientific). As positive control, zymosan (Sigma-Aldrich) was used at a final concentration of $0.5 \mathrm{mg} / \mathrm{mL}$.

\section{Determination of matrix metalloproteinase 9 (MMP-9) activities in PMN supernatants}

PMN $\left(10^{6} / 500 \mu \mathrm{L}\right.$ HBSS $\left./ 0.9 \mathrm{mM} \mathrm{CaCl} 2\right)$ were exposed to equal numbers of $E$. bovis sporozoites (15 and $30 \mathrm{~min}$, $\left.37^{\circ} \mathrm{C}\right)$. Stimulation of PMN with PAF (100 nM, $5 \mathrm{~min}$, $37^{\circ} \mathrm{C}$ ) was used for positive controls. PMN cultivated in plain medium were used for negative controls. After incubation, the cells were centrifuged $(600 \times g, 6 \mathrm{~min})$ and the supernatants were assayed for gelatinase activity by zymography. Therefore, substrate gel electrophoresis was performed using the method described by Conejeros et al. [32]. Briefly, $10 \mu \mathrm{L}$ of supernatant/slot were loaded on polyacrylamide gels $(10 \%, 0.75 \mathrm{~mm}$ thickness) containing $0.28 \%$ of gelatin (Sigma-Aldrich). In parallel a recombinant MMP-9 standard (Sigma-Aldrich) and a molecular mass marker (Fermentas International Inc., Canada) were loaded as reference samples. The gels were run at $200 \mathrm{~V}$ for $1 \mathrm{~h}$ in a Bio-Rad Mini Protean II chamber (Bio-Rad Laboratories, CA, USA). Thereafter, the gels were incubated twice in Triton X-100 (2.5\%, under constant shaking, RT, $30 \mathrm{~min}$ ) and overnight at $37^{\circ} \mathrm{C}$ in reaction buffer (100 mM Tris, pH 7.5; $\left.10 \mathrm{mM} \mathrm{CaCl}_{2}\right)$. The gels were stained in Coomassie Brilliant Blue R-250 (Winkler, Santiago, Chile; $0.5 \%$ in acetic acid : methanol $:$ water $=1$ : $3: 6$ ). MMP-9 (gelatinase B) enzymatic activity present in the test samples were determined according to the degree of gelatin degradation (visible as clear bands of $82 \mathrm{kDa}$ ) relative to the MMP-9-control by means of band intensity measurements (applying ImageJ $1.35 \mathrm{~s}$ software).

\section{Host cell infection and production of viable Eimeria bovis merozoites I}

Primary bovine umbilical vein endothelial cells (BUVEC) were isolated from umbilical cord veins, according to the method of Hermosilla et al. [4]. Confluent BUVEC monolayers $(n=3)$ were infected with $2.5 \times 10^{5}$ freshly excysted E. bovis-sporozoites suspended in modified ECGM [ECGM (PromoCell) supplemented with 70\% (v/v) M199 (Gibco), 2\% FCS (Gibco) and 1\% penicillin (SigmaAldrich)] per $25 \mathrm{~cm}^{2}$ flask (Greiner). The cells were fed $24 \mathrm{~h}$ after infection and thereafter every third day with modified ECGM. To control for E. bovis macromeront development and the release of merozoites I, infected BUVEC cultures were analyzed daily over a period of 22 days using a phase-contrast inverted microscope (IX81 microscope ${ }^{\circ}$, Olympus). Free-released merozoites I were harvested from the supernatant of infected BUVEC monolayers $(400 \times g, 5 \mathrm{~min})$ and washed twice in PBS. The merozoites I were counted in a Neubauer chamber haemocytometer.

\section{Scanning electron microscopy (SEM)}

Bovine PMN were incubated with E. bovis sporozoites, merozoites I or oocysts at a ratio of $1: 1$ for $90 \mathrm{~min}$ on poly-L-lysine (Sigma-Aldrich) pre-coated coverslips. After incubation, the samples were fixed (2.5\% glutaraldehyde in $0.1 \mathrm{M}$ cacodylate buffer, $15 \mathrm{~min}$, all Merck) and washed with $0.1 \mathrm{M}$ cacodylate buffer. The samples were post-fixed in $1 \%$ osmium tetroxide (Merck) in $0.1 \mathrm{M}$ cacodylate buffer, washed three times in distilled water, dehydrated in ascending ethanol concentrations, critical point dried by $\mathrm{CO}_{2}$-treatment and sputtered with gold. Specimens were examined using a Philips XL30 scanning electron microscope at the Institute of Anatomy and Cell Biology, Justus Liebig University Giessen, Germany.

\section{Quantification of NET derived from PMN and parasites from different origins}

NET formation was quantified using Pico Green ${ }^{\circ}$ (Invitrogen), a DNA-binding dye. In order to quantify $E$. bovis sporozoite- or merozoite I-induced NET formation, bovine PMN ( $n=3-5)$ were re-suspended in serum-free medium RPMI 1640 and incubated (all at $\left.37{ }^{\circ} \mathrm{C}\right)$ with E. bovis-sporozoites in a $1: 2$ ratio $\left(2 \times 10^{5}\right.$ PMN: $4 \times 10^{5}$ sporozoites, $\left.60 \mathrm{~min}\right)$, with $E$. bovis-merozoites I in a 1:1 ratio $\left(1 \times 10^{5} \mathrm{PMN}: 1 \times 10^{5}\right.$ merozoites, $\left.90 \mathrm{~min}\right)$, with oocysts $\left(5 \times 10^{4}, 60 \mathrm{~min}, 1: 1\right)$ and with sporozoites of $E$. arloingi ( $n=3 ; 1 \times 10^{5}$ PMN: $1 \times 10^{5}$ sporozoites, $\left.60 \mathrm{~min}\right)$, Isospora suis $\left(n=3 ; 1 \times 10^{5}\right.$ PMN: $1 \times 10^{5}$ sporozoites; $60 \mathrm{~min})$ and $T$. gondii $\left(n=5 ; 1 \times 10^{5} \mathrm{PMN}: 1 \times 10^{5}\right.$ sporozoites; $60 \mathrm{~min}$ ). In addition, PMN isolated from different host species origin (caprine, horse and dog) were incubated with E. bovis sporozoites in a $1: 2$ ratio $\left(2 \times 10^{5} \mathrm{PMN}: 4 \times 10^{5}\right.$ sporozoites, $60 \mathrm{~min}$ at $37{ }^{\circ} \mathrm{C}$ ). For positive controls, zymosan was used at a final concentration of $0.5 \mathrm{mg} / \mathrm{mL}$. Plain RPMI 1640 medium served as negative controls. NET-formation was determined based on Pico Green -DNA staining as described above. 


\section{Eimeria bovis sporozoite entrapment assay}

For the quantification of sporozoite entrapment within NET structures we followed the method described by Köckritz-Blickwede et al. [8] with slight modifications. Briefly, PMN $\left(2.5 \times 10^{5} / 96\right.$-well $)$ were pre-activated by zymosan treatment $\left(30 \mathrm{~min}, 37^{\circ} \mathrm{C}\right)$. Meanwhile, E. bovissporozoites were stained with FITC dye $(0.2 \mathrm{mg} / \mathrm{mL}$, $30 \mathrm{~min}$, on ice, in the dark, Invitrogen) and washed twice in PBS $(3000 \times g, 10 \mathrm{~min})$. Thereafter, zymosanstimulated PMN were exposed to FITC-labeled E. bovis sporozoites $(512 \times g, 10 \mathrm{~min}$; subsequent incubation for $30 \mathrm{~min}$ at $37^{\circ} \mathrm{C}$ ) in ascendant ratios $(0.5: 1 ; 1: 1$ and $1: 2)$. Non-exposed sporozoites were used for controls (1:1; 1:2). The samples were washed twice in RPMI 1640 medium and measured for fluorescence intensity at 485/ $538 \mathrm{~nm}$ wavelength. The percentage of entrapment was calculated as follows: [(A485/538 nm sporozoites exposed to $\mathrm{PMN}) /(\mathrm{A} 485 / 538 \mathrm{~nm}$ non-exposed sporozoites)] $\times 100 \%$.

\section{Ethics statement}

All animal procedures were performed according to the Justus Liebig University Animal Care Committee guidelines, approved by the Ethic Commission for Experimental
Animal Studies of the State of Hesse (Regierungspräsidium Giessen) and in accordance to the current German Animal Protection Laws. Identification number of animal care and project licence: GI 18/10-Nr.A51/2012 544_AZ952 (Eimeria bovis-oocyst production); GI 18/10Nr.A9/2012 521_AZ877 (Bovine blood samples). European Animal Welfare Legislation: ART13TFEU.

\section{Statistical analysis}

Co-culture/stimulation conditions were compared by oneor two-factorial analyses of variance (ANOVA) with repeated measurements in order to compare co-culture/stimulation conditions. All analyses were performed with the GraphPad Prism $^{\bullet} 6$ software. Differences were regarded as significant at a level of $\left.p \leq 0.05{ }^{(*)}\right) ; p \leq 0.01\left(^{* * *}\right) ; p \leq 0.001{ }^{(* * *)}$.

\section{Results}

Exposure to $E$. bovis sporozoites up-regulates neutrophil CD11b surface expression on bovine PMN and triggers NET formation in a CD11b-dependent manner

Since no data are available on PMN-derived receptors being involved in E. bovis-mediated NETosis, we here analyzed whether sporozoite exposure would affect CD11b
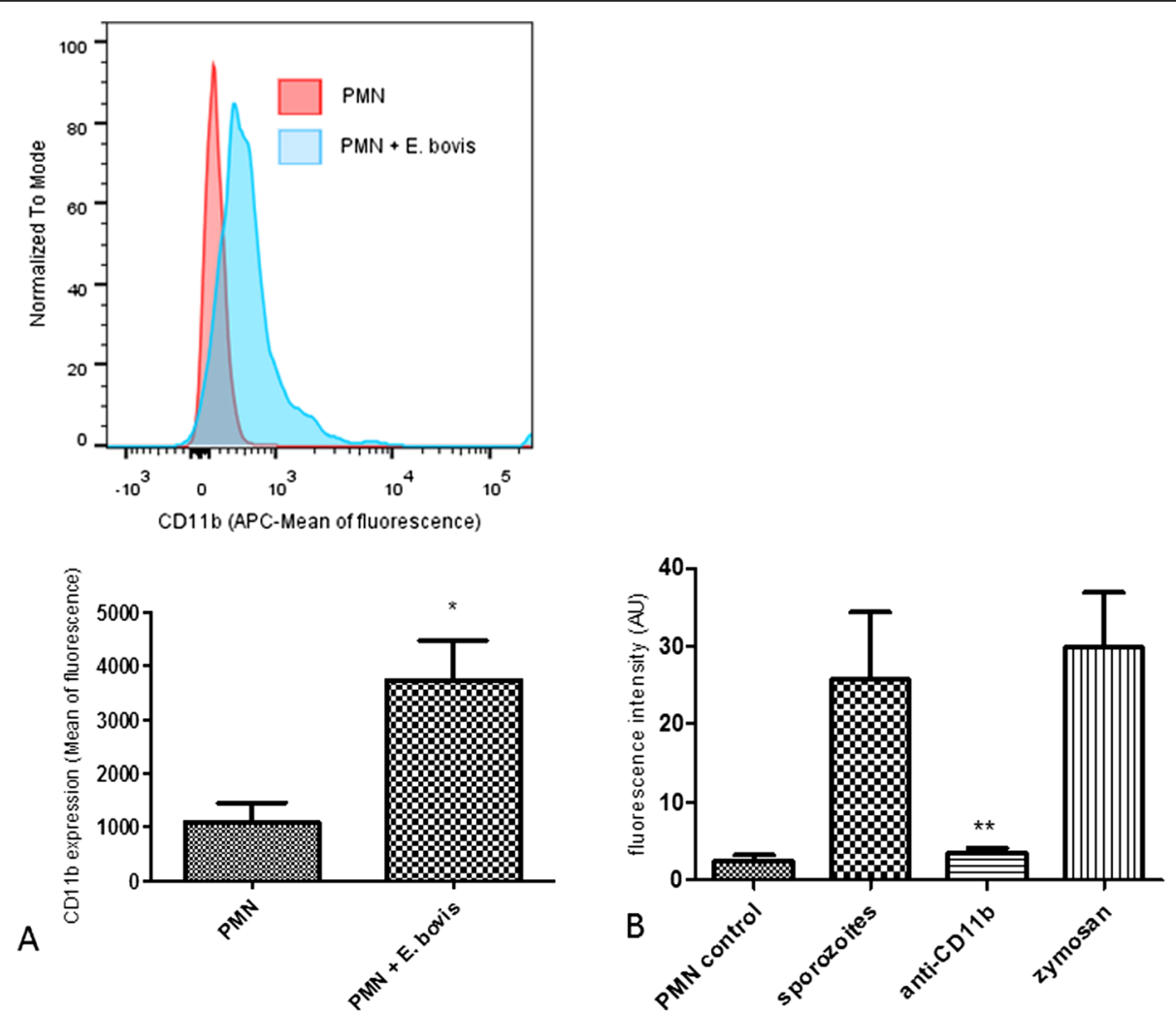

Figure $1 \mathrm{CD} 11 \mathrm{~b}$ surface expression and CD11b-dependent-NET formation of bovine $E$. bovis sporozoite-exposed PMN. (A) Bovine PMN were exposed to CFSE-labeled sporozoites (1:1) and subjected to FACS analysis probing with an antibody directed against CD11b. Non-exposed PMN in plain medium served as negative controls. The histograms represent the shift in the mean fluorescence of the PMN population analyzed and are representative of the experiments performed. (B) NET formation in the presence of anti-CD11b antibodies. Bovine PMN were pre-treated with anti-CD11b prior to sporozoite exposure. Stimulation with zymosan served as a positive control; PMN in plain medium were used for negative controls. 


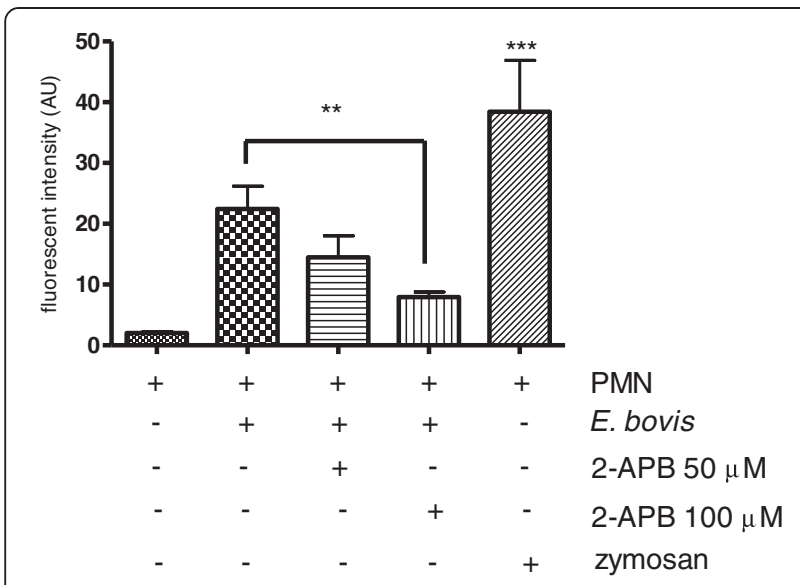

Figure 2 Influence of store-operated calcium entry (SOCE) on E. bovis sporozoites-induced NET formation. PMN were treated with the SOCE-inhibitor 2-APB prior to exposure to E. bovis sporozoites. For negative controls, non-treated PMN were used. For positive controls, zymosan stimulation was used. NET formation was quantified based on DNA-derived fluorescence intensities using an automate plate reader.

surface expression of bovine PMN. Applying FACS methodology, a significant parasite-triggered increase of CD11b surface expression was shown when compared to non-exposed PMN ( $p \leq 0.05$, Figure 1A). In addition, blockage of CD11b via specific antibodies led to a significant reduction in sporozoite-mediated NET formation when compared to antibody-free controls $(p \leq 0.01$;
Figure 1B). We also did not observe any significant NET induction using our irrelevant antibody control. Thus, these results suggest CD11b as a potential candidate for neutrophil receptors being involved in NET formation.

\section{Eimeria bovis-induced NET formation is store-operated calcium entry (SOCE)-dependent}

As previously shown in the bovine system, ROS production is a $\mathrm{Ca}^{2+}$-dependent process [32]. Since sporozoite-triggered NETosis was recently proven to be ROS-dependent [5], we here analyzed whether the presence of the $\mathrm{Ca}^{2+}$ (SOCE) inhibitor 2-APB [31,32] would influence sporozoite-mediated NETosis. The fact that treatment with $100 \mu \mathrm{M} 2-\mathrm{APB}$ resulted in a significant reduction of $E$. bovis sporozoiteinduced NET when compared with non-treated but sporozoite-exposed PMN ( $p \leq 0.01$, Figure 2$)$, suggests a $\mathrm{Ca}^{2+} / \mathrm{SOCE}$-dependent process. Overall, stimulation of PMN with zymosan revealed to be a potent SOCE inducer compared to the negative control ( $p \leq 0.001$; Figure 2).

\section{Sporozoite-induced NETosis is a ROS-, NE- MPO- dependent process}

Experiments applying specific antibodies against $\mathrm{H} 3, \mathrm{NE}$ and MPO alongside with DNA-staining proved NET structures (estimated via extracellular DNA-positive fibres) as co-localizing with histone H3-, NE- and MPO-positive signals indicated a pivotal role of these molecules in
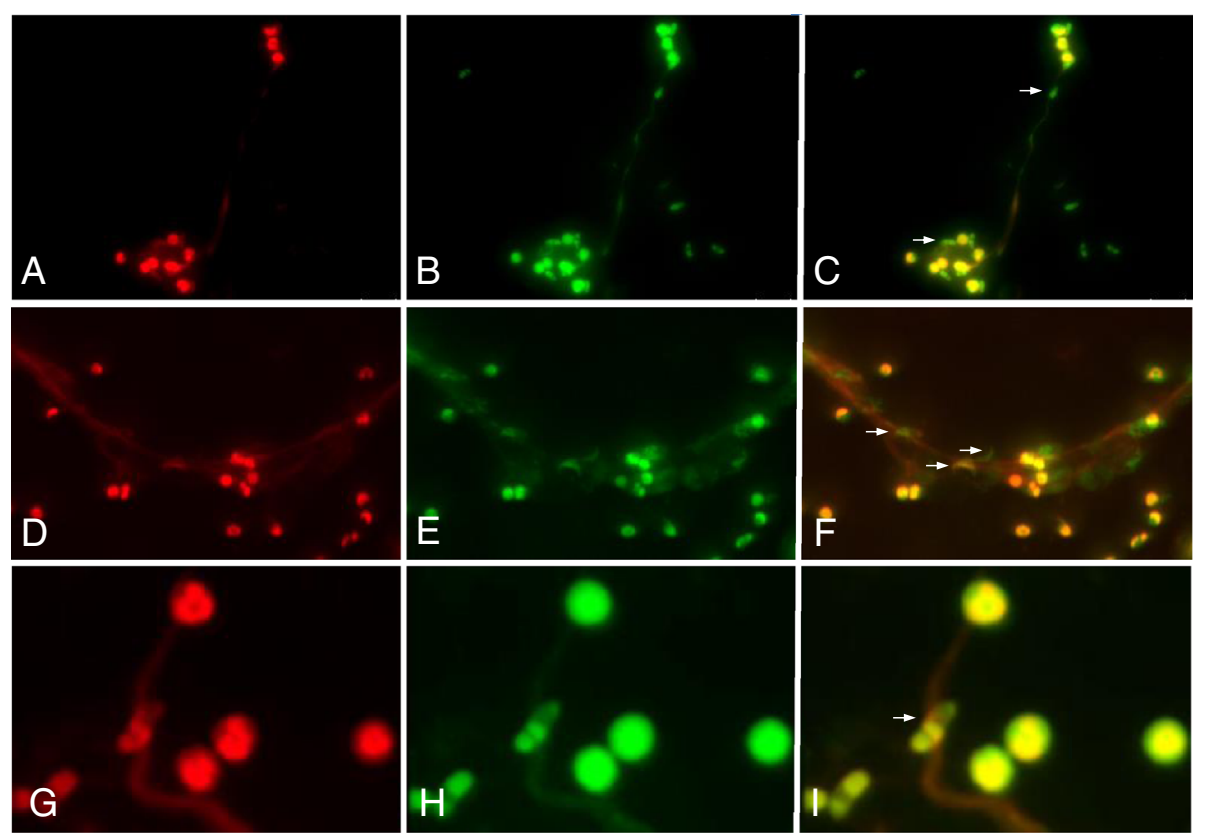

Figure 3 Co-localization of DNA with histones (H3), neutrophil elastase (NE) and myeloperxodase (MPO) in E. bovis sporozoite-induced NET structures. Co-cultures of bovine PMN and E. bovis sporozoites and oocysts were fixed, permeabilized and stained for DNA using Sytox Orange (red: A, D, G) and probed for histone $3(\mathrm{H} 3)$ (green: B), MPO (green: E) and NE (green: $\mathbf{H}$ ) using anti-H3, anti-NE and anti-MPO antibodies jointly with adequate conjugate systems. Areas of respective co-localization (merges) are illustrated in $\mathbf{C}, \mathbf{F}$, I. Photomicrographs are of representative cells from 3 independent experiments. 
sporozoite-triggered NETosis and confirmed classical NET characteristics (Figure 3). In addition, PMN exposure to $E$. bovis sporozoites resulted in a significant up-regulation of NADPH oxidase-, NE- and MPO- enzymatic activities $(p \leq 0.05$ for NADPH oxidase; $p \leq 0.01$ for NE and MPO; Figures $4 \mathrm{~A}-\mathrm{C})$. Furthermore, we confirmed the key role of these enzymes since inhibition of NADPH oxidase, NE and MPO led to significantly reduced sporozoite-triggered NETosis when compared to non-treated controls (Figure 4D; DPI and ABAH: $p \leq 0.05$; CMK: $p \leq 0.01$ ). As expected, DNase I treatments led to a significant NET resolution $(p \leq 0.001$, Figure 4D). Total DNA release from PMN was controlled by Triton $\mathrm{X}$ treatments revealing a proportion of up to $43 \%$ of PMN to be involved in sporozoite-mediated NETosis. Overall, these data confirm the relevance of $\mathrm{NADPH}$ oxidase, NE and MPO in E. bovis sporozoiteinduced NET formation.

\section{$E$. bovis sporozoite exposure enhances matrix} metalloprotease 9 (MMP-9) release of bovine PMN

Given that tertiary granule contents of PMN also contribute to NET formation based on their antimicrobial peptide/protease activities as described by Brinkmann et al. [6], we additionally analyzed PMN supernatants for the enzymatic activity of MMP-9. Co-cultures of PMN with $E$. bovis sporozoites led to a significant increase of MMP-9 release $(p \leq 0.05$; Figure 5$)$ at both time points tested (15 and $30 \mathrm{~min}$ ) indicating that tertiary granules or MMP-9 may also be involved in sporozoite-triggered NETosis. As expected, stimulation with PAF revealed to be a reliable positive control for MMP-9 release in the bovine system $(p \leq 0.05)$.

\section{ERK1/2- and p38 MAPK-signaling pathways are involved}

\section{in $E$. bovis sporozoite-triggered NET extrusion}

We analyzed whether the ERK1/2 and p38 MAPK signaling pathway is involved in sporozoite-triggered NETosis. Exposure of E. bovis sporozoites to PMN resulted in a fast and significant phosphorylation of ERK1/2 ( $p \leq 0.05$ after 15 and $30 \mathrm{~min}$ of incubation, Figure 6A) and p38 ( $p \leq 0.05$ at $15 \mathrm{~min}$, Figure 6B) MAPK. Furthermore, experiments applying inhibitors of ERK1/2 and p38 MAPK confirmed the relevance of this signaling pathway since treatments with both inhibitors resulted in a significant reduction of sporozoite-mediated NET formation (UO126: $p \leq 0.01$ and SB 203580: $p \leq 0.05$, Figure 6C).

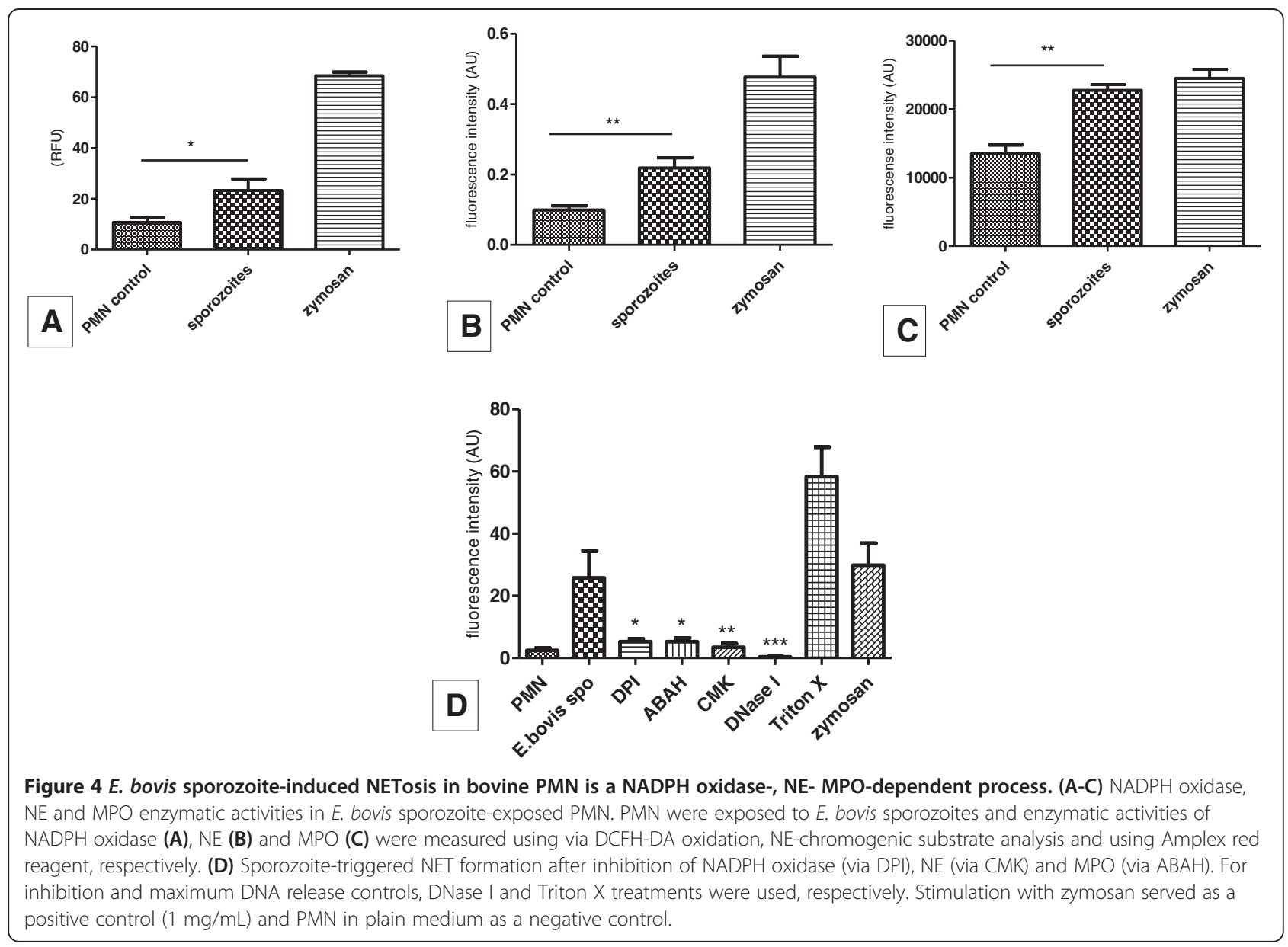




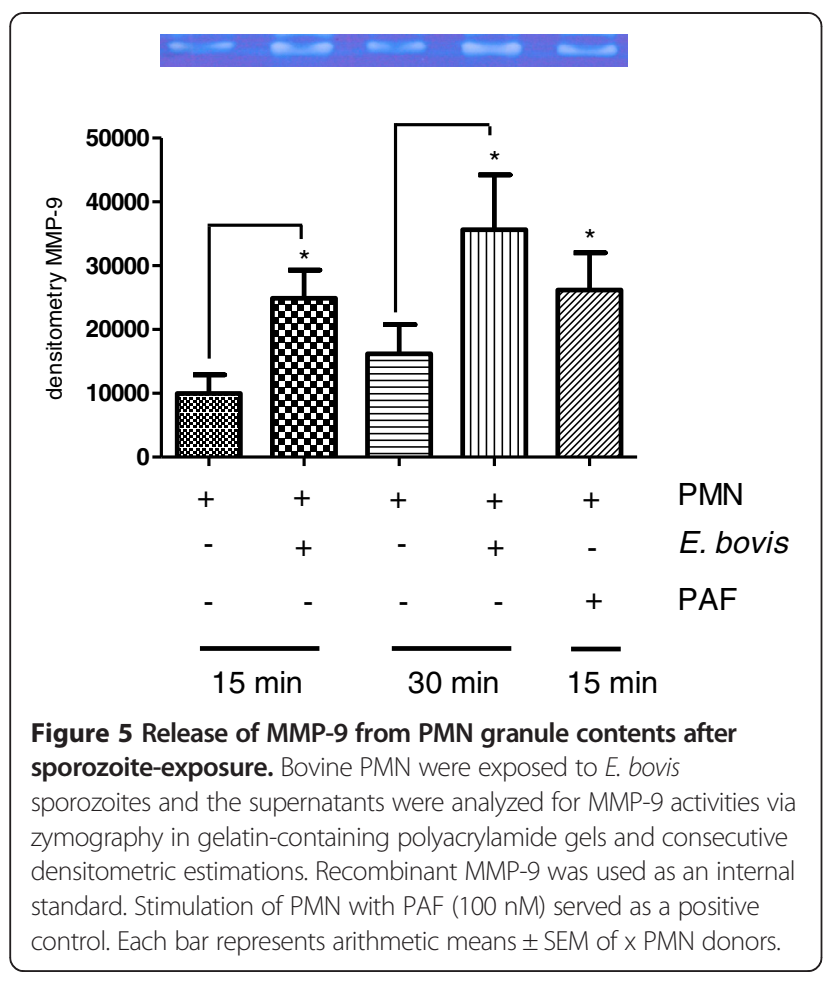

\section{Sporozoite-triggered NETosis is neither stage- nor parasite} species- nor host-specific

To account for stage-specificity we tested E. bovis merozoites I for the capability to induce NET. As depicted in Figure $7 \mathrm{C}$, merozoite I stages significantly triggered NET when compared to non-exposed controls $(p \leq 0.01)$. These data were confirmed by SEM analyses illustrating the formation of a delicate, NET-like network of thicker and thinner strands of fibres originating from dead PMN and being firmly attached to the merozoites I (Figure 7A). In addition, SEM analyses indicated NET-like structures also to be induced by oocyst stages (Figure 7B). These data clearly argue against a stage-specificity of E. bovis-triggered NETosis highlighting the capability of PMN to equally respond to different parasitic stages of this parasite.

In order to analyze whether E. bovis-triggered NETosis is a parasite-specific event or rather reflects a general mechanism that commonly accounts for most coccidian parasites, we analyzed NET formation of bovine PMN after exposure to the sporozoite stage of a non-bovine Eimeria spp. (the caprine-specific species E. arloingi) and of two non-Eimeria coccidian species ( $T$. gondii and $I$. suis). The data clearly show that the sporozoites of all three parasite species equally induced significant NET formation (E. arloingi and T. gondii: $p \leq 0.01$, I. suis: $p \leq 0.05$, Figure 8B). Overall, these findings rather argue against a parasite-specific mechanism and propose NET formation as a generally valid effector mechanism against the sporozoite stage of different coccidian species.
Given that $E$. bovis is a strict host-specific parasite and, in consequence, exclusively infects bovines, we then analyzed whether $E$. bovis-mediated NET formation is a host-specific event. Therefore, PMN of different host origins (horse, goat, dog and cattle) were controlled for NET formation after exposure to $E$. bovis sporozoites. The data clearly revealed that PMN of all host types reacted by significant NET formation ( $p \leq 0.01$, Figure 8A) which argues against a host-specific reaction.

\section{NET structures significantly entrap Eimeria bovis sporozoites}

In order to analyze the capacity of NET to trap viable $E$. bovis sporozoites, we established a quantitative parasiteentrapment-assay by using FITC-stained parasites and zymosan pre-activated bovine PMN as described elsewhere [17]. The experiments revealed a proportion of $43.4 \%$ of sporozoites to be immobilized in NET structures when compared to non-exposed sporozoites (data not shown). These results indicate a rather high efficacy of NET as an effector mechanism considering that almost every second parasite was entrapped and most probably hampered from host cell invasion as reported elsewhere [5].

\section{Discussion}

NET formation depends on the assembly/activation of the NADPH oxidase complex and the resulting production of ROS [7]. Since these mechanisms were reported as $\mathrm{Ca}^{2+}$-mediated in the bovine system [31], it appears likely that NETosis is a $\mathrm{Ca}^{2+}$-dependent process, as recently demonstrated by Gupta et al. [20]. The fact that NET formation is induced by thapsigargin [33] which mobilizes $\mathrm{Ca}^{2+}$ from intracellular pools, also indicates a key role of $\mathrm{Ca}^{2+}$ in NETosis. Since SOCE was proven to play a crucial role in $\mathrm{Ca}^{2+}$-dependent $\mathrm{ROS}$ production [34] we here used 2-APB a well-known inhibitor of SOCE for functional assays. The pre-incubation of PMN with 2-APB prior to sporozoite exposure resulted in a significant diminishment of NETosis and therefore confirmed the assumption that parasite-triggered NET formation is a $\mathrm{Ca}^{2+}$-(SOCE)-dependent process.

Besides being SOCE-dependent, ROS production is upregulated in a CD11b (CR3)-dependent manner in bovine PMN [31]. In turn, up-regulation of CD11b proves as SOCE-dependent since it is inhibited by 2-APB [32] leading us to the assumption that the neutrophil integrin receptor CD11b may also be involved in sporozoite-mediated NETosis. Indeed, we found significantly enhanced levels of surface CD11b expression on parasite-exposed bovine PMN. The fact that antibody-mediated blockage of CD11b additionally resulted in NET diminishment suggests CD11b to be involved in sporozoite-triggered NETosis and to function as a PMN-derived receptor in this effector mechanism. These findings were in accordance 

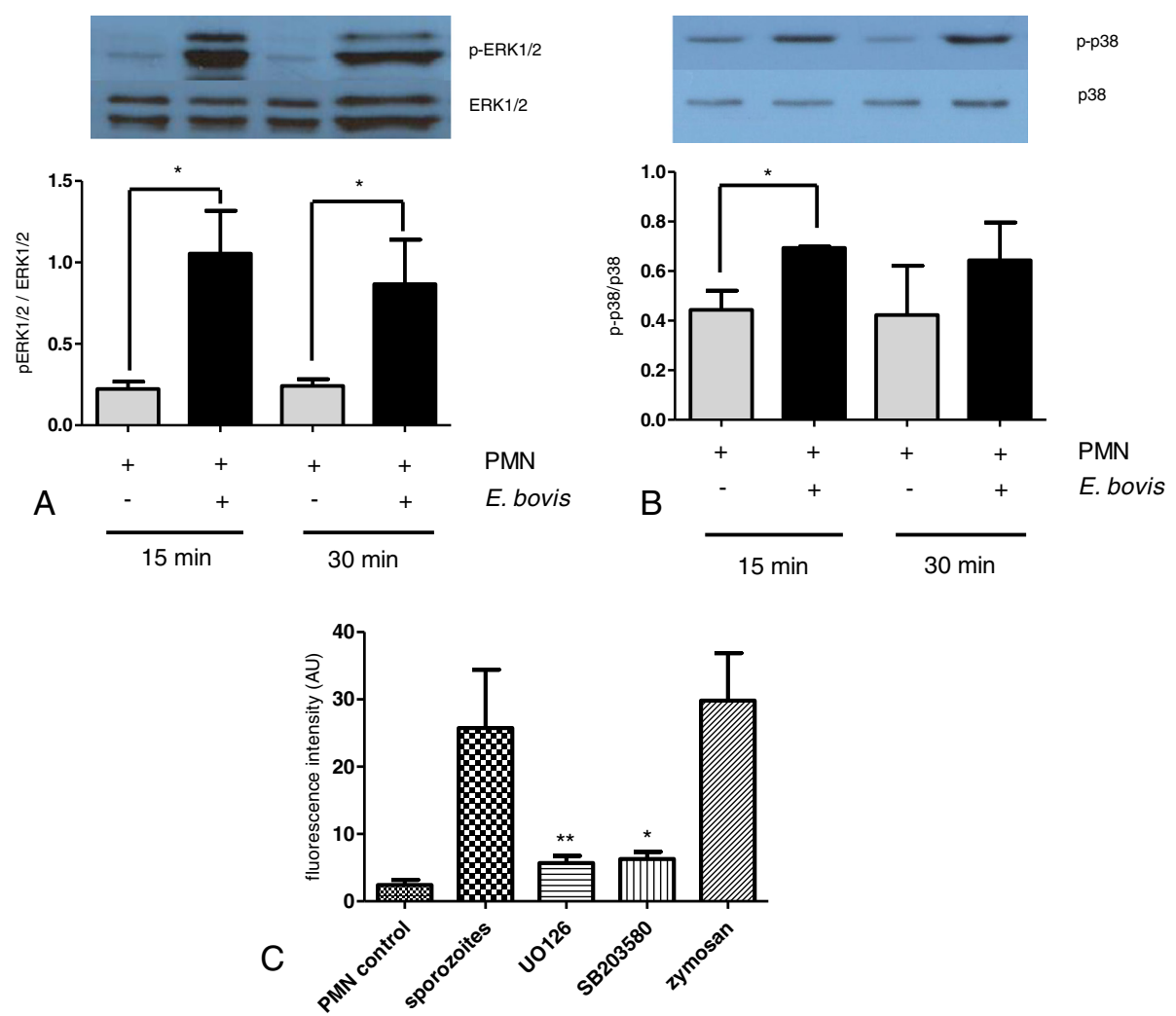

Figure 6 Expression of phosphorylated ERK1/2- and p38 MAPK in sporozoite-exposed PMN and influence of their inhibition on parasite-triggered NETosis. Total proteins of sporozoite-exposed PMN and non-exposed controls were analyzed by SDS/PAGE and immunoblot technique using specific antibodies against non-phosphorylated and phosphorylated ERK1/2 (A) and p38 MAPK (B). Respective bands were densitometrically analyzed. Each bar represents the arithmetic means \pm SEM of three PMN donors. Differences were regarded as significant at a level of $p \leq 0.05$ compared to negative controls. (C) NET formation in presence of ERK 1/2 (UO126) and p38 (SB 203580) MAPK inhibitors. Stimulation with zymosan served as a positive control; PMN in plain medium as a negative control.

with Byrd et al. [21] who reported on CD11b as a potential ligand in NETosis since antibody-mediated blockage of this integrin inhibits fibronectin-dependent NET formation to C. albicans hyphae.

Moreover, SOCE is tightly regulated via ERK1/2 MAPK phophorylation, i.e., by a signaling pathway that was recently described to be involved in NETosis [35]. In addition, ROS-dependent activation of ERK and p38 MAPK was demonstrated to mediate PMA-induced NET release from human neutrophils [35]. In accordance to the latter reports and to data on other pathogens $[12,21]$ we here show a sporozoite-triggered up-regulation of ERK1/2 and p38 MAPK phosphorylation in parasiteexposed bovine PMN. The key role of the ERK1/2- and p38 MAPK-dependent signaling pathway in NETosis was confirmed in functional inhibition experiments showing that parasite-triggered NETosis was significantly diminished in the presence of ERK-and p38 inhibitors.

We furthermore focused on the relevance of $\mathrm{NE}$ and MPO and confirmed the key role of these two molecules in E. bovis-triggered NETosis. As with other bovine pathogens [18], exposure to sporozoites led to an increase of both, NE and MPO enzymatic activities in bovine PMN. Functional inhibition assays confirmed the relevance of these enzymes since parasite-mediated NETosis was significantly blocked when NE and MPO inhibitors were applied. Finally, the co-localization of DNA-rich filaments with NE- or MPO-derived signals in sporozoiteinduced NET structures emphasized the classical characteristics of NET. These results were in line with recent findings on other coccidian parasites, such as E. arloingi [16] or B. besnoiti [17].

Whilst NE and MPO originate from azurophilic PMN granules, other molecules such as metalloproteinases (MMP), are contained in tertiary (gelatinase) granules. We here analyzed whether MMP-9 is released from PMN upon sporozoite exposure. Zymographic analyses of PMN supernatants confirmed a parasite-mediated MMP-9-release into PMN supernatants. So far, the functional role of MMP-9 in NETosis is unclear. However, it is worth noting that the release of MMP-9 is regulated by the ERK $1 / 2$ and p38 MAPK signaling pathway [36]. Interestingly, Carmona Rivera et al. [37] showed an impaired endothelial function induced by NET-externalized 

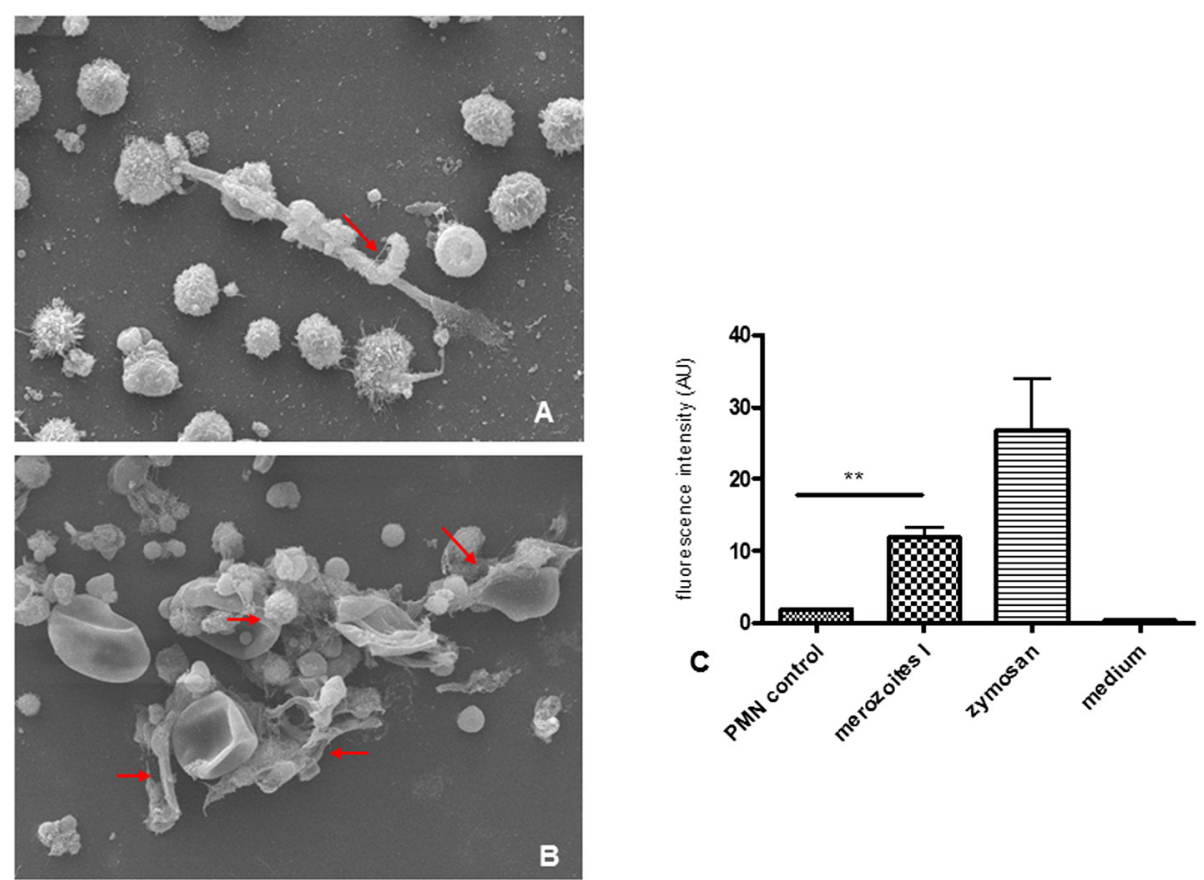

Figure 7 NET formation triggered by E. bovis merozoites I and oocyst stages. (A) Scanning electron microscopy (SEM) analysis of E. bovis merozoite l-exposed PMN. The arrow indicates a merozoite I being trapped in NET structures. (B) SEM analysis of E. bovis oocyst-exposed PMN revealing NET-like, delicate PMN-derived filaroid structures being attached to oocysts as indicated by arrows. (C) Quantification of E. bovis merozoite l-induced NET formation. PMN were exposed to E. bovis merozoites I, zymosan (positive control) or plain medium (negative control). NET formation was estimated by Pico Green-derived fluorescence intensities. Arithmetic means of three PMN donors, minimum and maximum.

MMP. Given that E. bovis sporozoites infect and mature within endothelial cells, a yet unclear interrelation between NETosis and parasite development within endothelium may exist.

Overall, since the first description of NET as an innate effector mechanism [6], most studies have focused on NETosis driven by bacterial, viral and fungal pathogens and, less frequently, on parasitic pathogens [13]. Nonetheless, there is increasing evidence on the relevance of NET as a defense mechanism against protozoan infections in vitro and in vivo [12,13]. Thus, NET-related data are available on some protozoan pathogens, such as E. bovis, Toxoplasma gondii, Plasmodium falciparum, E. arloingi, and B. besnoiti [5,12,15-17] and Leishmania spp. [14].

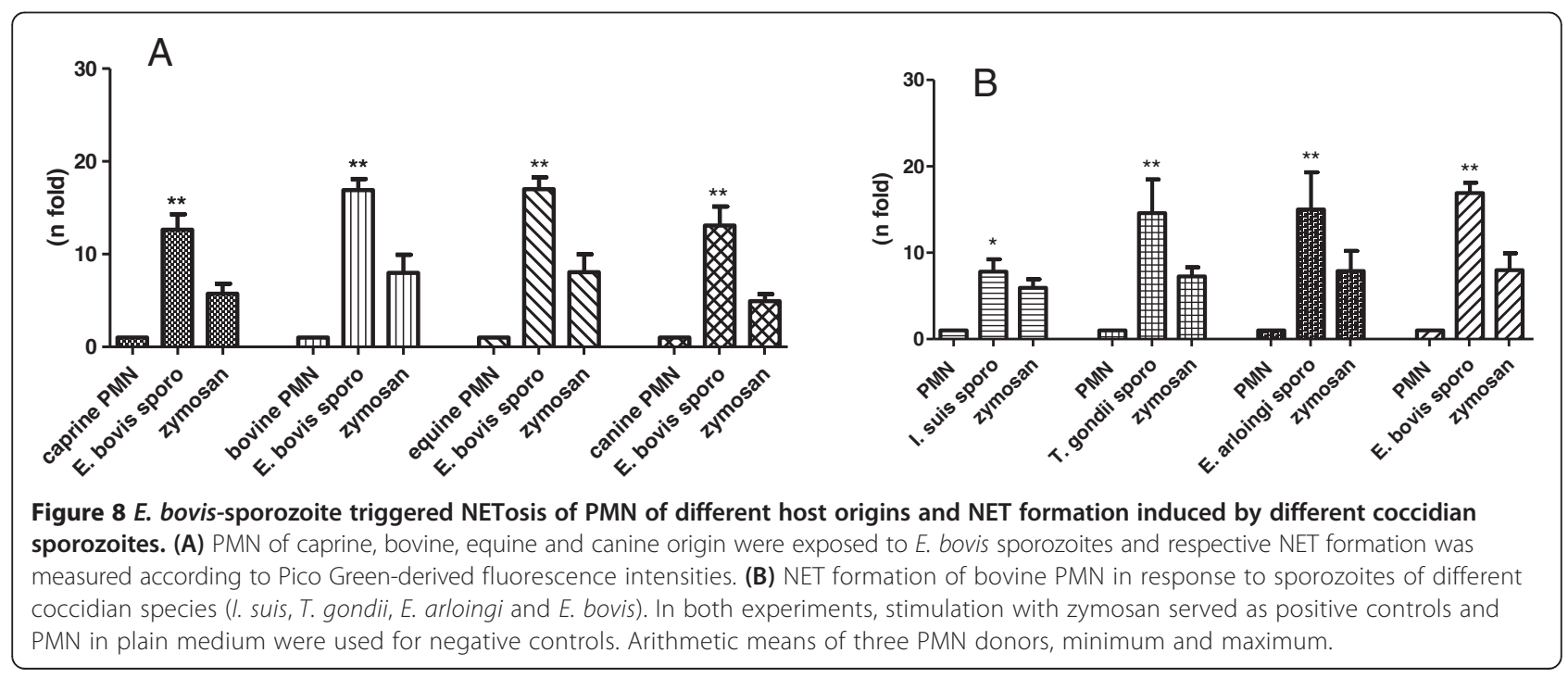


The current data suggest NETosis as a generally valid effector mechanism against coccidian parasites. Thus, NET release occurred irrespective of the parasite stage (E. bovis sporozoites, merozoites I, oocysts), and PMN origin (bovine, caprine, equine, canine). In agreement, data on different Leishmania stages [14] also indicated NETosis as a stage-independent defense mechanism. In addition, $T$. gondii-triggered NETosis has been reported for both human and murine PMN [12] indicating hostindependent reactions. Overall, it is noteworthy that the degree of entrapment clearly differed amongst different parasite species and stages. Thus, we here report on $43 \%$ of $E$. bovis sporozoites to be immobilized within bovine NET structures, whilst in the caprine system even $72 \%$ of $E$. arloingi sporozoites were trapped [16] but only $34 \%$ of $B$. besnoiti tachyzoites were found ensnared in NET being expelled from bovine PMN [17]. Since all these parasites exhibit an obligatory intracellular replication, extracellular immobilization via NET will have a tremendous implication on the outcome of the disease as previously postulated $[16,17]$.

Furthermore, NETosis was not parasite species-specific since sporozoites of different coccidian parasites $(E$. bovis, E. arloingi, I. suis, T. gondii) equally triggered NET formation. To our best knowledge this is the first report on $T$. gondii and I. suis sporozoite-induced NET extrusion whilst tachyzoite stages of T. gondii [12] and sporozoites of $E$. arloingi [16] have already been reported before. Especially in the case of $T$. gondii, which exhibits an enormous proliferation at the tachyzoite stage, the intervention of the immune system at a very early time point, i.e. when sporozoites invade the host system and before non-sexual parasite replication occurs, will have a higher impact on the outcome of disease than tachyzoitetriggered reactions. Taking into account that PMN have been demonstrated to actively transmigrate into the intestinal lumen and were found alive in the gut mucus [38], it seems feasible to assume that they may interact with luminal pathogen stages, such as ingested Eimeria oocysts, in vivo. Consistently, we here show that oocysts of E. bovis are trapped and sometimes almost encaged by NET structures in vitro, thereby most probably preventing proper excystation of sporozoites. This novel anti-excystation mechanism of NET has recently been hypothesized for $E$. arloingi oocysts, in which sporozoites were impeded to escape from NET-covered preformed excystation sites (micropyles) of sporulated oocysts [16]. Thus, in the in vivo situation, luminal occurring NETosis might intervene very early after oral infection with the parasite and abrogate infection even before infectious stages (sporozoites) are able to evade immune reactions via intracellular positioning.

In summary, NET formation seems to be an ancient and highly conserved host effector mechanism of PMN acting against several pathogens as an early host immune reaction. In the present work, we added new data on the molecular mechanisms involved in parasite-triggered NETosis and call for more investigations on receptorligand-interactions.

\section{Competing interests}

The authors declare that they have no competing interests.

\section{Authors' contributions}

Conceived and designed the experiments: TMC, JMH, IC, PA, RAB, MAH, CH, AT. Performed the experiments: TMC, JMH, IC, PA. Analyzed the data: TMC, $\mathrm{JMH}, \mathrm{IC}, \mathrm{PA}, \mathrm{RAB}, \mathrm{CH}, \mathrm{AT}$. Contributed reagents/materials/analysis tools: TMC, JMH, IC, PA, RAB, MAH, CH, AT. Wrote the paper: TMC, CH, AT. All authors read and approved the final manuscript.

\section{Acknowledgments}

We are deeply indebted to Gerd Magdowski (Institute of Anatomy and Cell Biology, JLU Giessen, Germany) for his excellent assistance and kind help on SEM analyses. We further acknowledge Brigitte Hofmann, Christin Ritter and Klaus Becker for their excellent technical assistance in cell culture and blood collection. This work was supported and financed by the German Research Foundation (DFG; grant number: TA 291/4-1).

\section{Author details}

${ }^{1}$ Institute of Parasitology, Justus Liebig University Giessen, Schubertstraße 81, 35392 Giessen, Germany. ${ }^{2}$ Laboratory of Inflammation Pharmacology, Institute of Pharmacology and Morphophysiology, Faculty of Veterinary Science, Austral University of Chile, Valdivia 5110566, Chile. ${ }^{3}$ Department of Biology, University of Nariño, Pasto 520002, Colombia.

Received: 10 September 2014 Accepted: 28 January 2015

Published online: 05 March 2015

\section{References}

1. Daugschies A, Najdrowski M (2005) Eimeriosis in cattle: current understanding J Vet Med B Infect Dis Vet Public Health 52:417-427

2. Friend SC, Stockdale PH (1980) Experimental Eimeria bovis infection in calves: a histopathological study. Can J Comp Med 44:129-140

3. Behrendt $\mathrm{H}$, Hermosilla C, Hardt M, Failing K, Zahner H, Taubert A (2008) PMN-mediated immune reactions against Eimeria bovis. Vet Parasitol 151:97-109

4. Hermosilla C, Zahner H, Taubert A (2006) Eimeria bovis modulates adhesion molecule gene transcription in and PMN adhesion to infected bovine endothelial cells. Int J Parasitol 36:423-431

5. Behrendt JH, Ruiz A, Zahner H, Taubert A, Hermosilla C (2010) Neutrophil extracellular trap formation as innate immune reactions against the apicomplexan parasite Eimeria bovis. Vet Immunol Immunopathol 133:1-8

6. Brinkmann V, Reichard U, Goosmann C, Fauler B, Uhlemann Y, Weiss DS, Weinrauch Y, Zychlinsky A (2004) Neutrophil extracellular traps kill bacteria. Science 303:1532-1535

7. Fuchs TA, Abed U, Goosmann C, Hurwitz R, Schulze I, Wahn V, Weinrauch Y, Brinkmann V, Zychlinsky A (2007) Novel cell death program leads to neutrophil extracellular traps. J Cell Biol 176:231-241

8. Köckritz-Blickwede M, Chow O, Ghochani M, Nizet V (2010) Visualization and functional evaluation of phagocyte extracellular traps. Method Microbiol 37:139-160

9. Guimarães-Costa AB, Nascimento MT, Wardini AB, Pinto-da-Silva LH, Saraiva EM (2012) ETosis: a microbicidal mechanism beyond cell death. J Parasitol Res 2012:929743

10. Brinkmann V, Zychlinsky A (2007) Beneficial suicide: why neutrophils die to make NETs. Nat Rev Microbiol 5:577-582

11. Brinkmann V, Zychlinsky A (2012) Neutrophil extracellular traps: is immunity the second function of chromatin? J Cell Biol 198:773-783

12. Abi Abdallah DS, Lin C, Ball CJ, King MR, Duhamel GE, Denkers EY (2012) Toxoplasma gondii triggers release of human and mouse neutrophil extracellular traps. Infect Immun 80:768-777

13. Hermosilla C, Munoz Caro T, Silva LM, Ruiz A, Taubert A (2014) The intriguing host innate immune response: novel anti-parasitic defence by neutrophil extracellular traps. Parasitology 141:1489-1498 
14. Guimarães-Costa AB, Nascimento MT, Froment GS, Soares RP, Morgado FN, Conceição-Silva F, Saraiva EM (2009) Leishmania amazonensis promastigotes induce and are killed by neutrophil extracellular traps. Proc Natl Acad Sci U S A 106:6748-6753

15. Baker VS, Imade GE, Molta NB, Tawde P, Pam SD, Obadofin MO, Sagay SA, Egah DZ, lya D, Afolabi BB, Baker M, Ford K, Ford R, Roux KH, Keller TC 3rd (2008) Cytokine-associated neutrophil extracellular traps and antinuclear antibodies in Plasmodium falciparum infected children under six years of age. Malar J 7:41

16. Silva LMR, Muñoz Caro T, Rüdiger G, Vila-Viçosa MJM, Cortes HC, Hermosilla T, Taubert A: (2014) The apicomplexan parasite Eimeria arloingi induces caprine neutrophil extracellular traps. Parasitol Res 113:2797-2807

17. Muñoz Caro T, Hermosilla C, Silva LMR, Cortes H, Taubert A (2014) Neutrophil extracellular traps as innate immune reaction against the emerging apicomplexan parasite Besnoitia besnoiti. PLoS One 9:e91415

18. Aulik NA, Hellenbrand KM, Klos H, Czuprynski CJ (2010) Mannheimia haemolytica and its leukotoxin cause neutrophil extracellular trap formation by bovine neutrophils. Infect Immun 78:4454-4466

19. Juneau RA, Pang B, Weimer KE, Armbruster CE, Swords WE (2011) Nontypeable Haemophilus influenzae initiates formation of neutrophil extracellular traps. Infect Immun 79:431-438

20. Gupta AK, Giaglis S, Hasler P, Hahn S (2014) Efficient neutrophil extracellular trap induction requires mobilization of both intracellular and extracellular calcium pools and is modulated by cyclosporine A. PLoS One 9:e97088

21. Byrd AS, O'Brien XM, Johnson CM, Lavigne LM, Reichner JS (2013) An extracellular matrix-based mechanism of rapid neutrophil extracellular trap formation in response to Candida albicans. J Immunol 190:4136-4148

22. Hermosilla C, Barbisch B, Heise A, Kowalik S, Zahner H (2002) Development of Eimeria bovis in vitro: suitability of several bovine, human and porcine endothelial cell lines, bovine fetal gastrointestinal, Madin-Darby bovine kidney (MDBK) and African green monkey kidney (VERO) cells. Parasitol Res 88:301-307

23. Pinckney RD, Lindsay DS, Toivio-Kinnucan MA, Blagburn BL (1993) Ultrastructure of Isospora suis during excystation and attempts to demonstrate extraintestinal stages in mice. Vet Parasitol 47:225-233

24. Freyre A, Falcón J (2004) Massive excystation of Toxoplasma gondii sporozoites. Exp Parasitol 107:72-77

25. Hidalgo MA, Ojeda F, Eyre P, LaBranche TP, Smith C, Hancke JL, Burgos RA (2004) Platelet-activating factor increases pH(i) in bovine neutrophils through the PI3K-ERK1/2 pathway. Br J Pharmacol 141:311-321

26. Hidalgo MA, Nahuelpan C, Manosalva C, Jara E, Carretta MD, Conejeros I, Loaiza A, Chihuailaf R, Burgos RA (2011) Oleic acid induces intracellular calcium mobilization MAPK phosphorylation, superoxide production and granule release in bovine neutrophils. Biochem Biophys Res Commun 409:280-286

27. Martinelli S, Urosevic M, Daryadel A, Oberholzer PA, Baumann C, Fey MF, Dummer R, Simon HU, Yousefi S (2004) Induction of genes mediating interferon-dependent extracellular trap formation during neutrophil differentiation. J Biol Chem 279:44123-44132

28. Hermosilla C, Stamm I, Taubert A, Lutz K, Zahner H, Menge C (2008) Fluorescent Eimeria bovis sporozoites and meront stages in vitro: a helpful tool to study parasite-host cell interactions. Parasitol Res 102:777-786

29. Scapinello S, Brooks AS, Maclnnes II, Hammermueller J, Clark ME, Caswell J (2011) Bactericidal activity of porcine neutrophil secretions. Vet Immunol Immunopathol 139:113-118

30. Parker H, Albrett AM, Kettle AJ, Winterbourn CC (2012) Myeloperoxidase associated with neutrophil extracellular traps is active and mediates bacterial killing in the presence of hydrogen peroxide. J Leukoc Biol 91:369-376

31. Conejeros I, Patterson R, Burgos RA, Hermosilla C, Werling D (2011) Induction of reactive oxygen species in bovine neutrophils is CD11b, but not dectin-1-dependent. Vet Immunol Immunopathol 139:308-312

32. Conejeros I, Jara E, Carretta MD, Alarcón P, Hidalgo MA, Burgos RA (2012) 2Aminoethoxydiphenyl borate (2-APB) reduces respiratory burst, MMP-9 release and CD11b expression, and increases I-selectin shedding in bovine neutrophils. Res Vet Sci 92:103-110

33. Gupta AK, Joshi MB, Philippova M, Erne P, Hasler P, Hahn S, Resink TJ (2010) Activated endothelial cells induce neutrophil extracellular traps and are susceptible to NETosis-mediated cell death. FEBS Lett 584:3193-3197

34. Hallett MB, Davies EV, Campbell AK (1990) Oxidase activation in individual neutrophils is dependent on the onset and magnitude of the Ca2+ signal. Cell Calcium 11:655-663
35. Hakkim A, Fuchs TA, Martinez NE, Hess S, Prinz H (2011) Activation of the Raf-MEK-ERK pathway is required for neutrophil extracellular trap formation. Nat Chem Biol 7:75-77

36. Wang $X$, Mori $T$, Jung JC, Fini ME, Lo EH (2002) Secretion of matrix metalloproteinase-2 and -9 after mechanical trauma injury in rat cortical cultures and involvement of MAP kinase. J Neurotrauma 19:615-625

37. Carmona-Rivera C, Zhao W, Yalavarthi S, Kaplan MJ. Neutrophil extracellular traps induce endothelial dysfunction in systemic lupus erythematosus through the activation of matrix metalloproteinase-2. Ann Rheum Dis. in press.

38. Sumagin R, Robin AZ, Nusrat A, Parkos CA (2014) Transmigrated neutrophils in the intestinal lumen engage ICAM-1 to regulate the epithelial barrier and neutrophil recruitment. Mucosal Immunol 7:905-915

\section{Submit your next manuscript to BioMed Central and take full advantage of:}

- Convenient online submission

- Thorough peer review

- No space constraints or color figure charges

- Immediate publication on acceptance

- Inclusion in PubMed, CAS, Scopus and Google Scholar

- Research which is freely available for redistribution

Submit your manuscript at www.biomedcentral.com/submit 